\title{
Ciclo de Mejora Docente en la Parte Práctica de la Asignatura de Proyectos. Anteproyecto de una Fábrica de Cerveza
}

Miguel ÁNGEL ZARCO GARRIDO

Universidad de Sevilla

Departamento de Ingeniería de la

Construcción y Proyectos de Ingeniería

mzarco@us.es

ORCID: https://orcid.org/0000-0002-1455-9468

D.O.I.: http://dx.doi.org/10.12795/JDU.2018.i01.58

Pp.: 1035-1052

\section{Resumen}

La experimentación docente universitaria que he llevado a cabo en el Segundo Ciclo de Mejora del Curso General de Docencia Universitaria 5 (CGDU5) ha tenido lugar en la Parte Práctica de la asignatura de Proyectos del cuarto curso de las titulaciones de Grado en Ingeniería de Tecnologías Industriales (GITI) y Grado en Ingeniería Química (GIQ) que se imparten en la Escuela Técnica Superior de Ingenieros de la Universidad de Sevilla, durante el primer cuatrimestre del curso académico $2018 / 2019$.

En la Parte Práctica de esta asignatura se lleva a cabo el desarrollo y la redacción (en grupo) de un proyecto de Ingeniería Industrial con un alcance similar a un anteproyecto o proyecto básico. 
Palabras Clave: Proyectos, Grado en Ingeniería de Tecnologías Industriales, Grado en Ingeniería Química, Docencia Universitaria, Experimentación Docente Universitaria.

\section{Descripción del Contexto}

Teniendo en cuenta que los alumnos (20) son de dos Grados (GITI y GIQ), con distintas especialidades (Química Industrial, Organización y Producción, Energética, Mecánica-Máquinas, Eléctrica, Electrónica, Automática y Procesos químicos), el anteproyecto debe tener contenido para todas ellas; ya que el trabajo de la asignatura, que se realiza en grupo, se realiza a su vez en subgrupos, tantos como especialidades hay en clase, y cada uno de ellos desarrolla y redacta la parte del anteproyecto propia de su especialidad.

El proyecto a desarrollar lo eligen los alumnos y en el caso que nos ocupa han elegido redactar el anteproyecto de una fábrica de cerveza.

La asignatura la enfoco como si estuviese en la vida laboral real. Como si yo tuviese que redactar un anteproyecto que me han encargado (el que han elegido los alumnos) y que el equipo de gente del que dispongo para realizarlo son los alumnos. Intento que ellos se impliquen desde el primer momento ya que son los que van a realizarlo. El trabajo del anteproyecto lo hacen los alumnos fuera del horario de clase trabajando individualmente e interactuando en los grupos por especialidad según el caso y momento. Las clases equivaldrían a las reuniones de trabajo de seguimiento (reales) del equipo de redacción del anteproyecto.

\section{Diseño previo del Ciclo de Mejora Docente}

En el Ciclo de Mejora Docente se trabajarán aquellas partes del anteproyecto que se estén redactando en ese momento. Se trata del Documento Planos, del Documento Presupuesto y de los Anejos a la Memoria: Programa de Trabajos, Seguridad y Salud y Control de Calidad. 


\section{Mapa de contenidos y problemas}

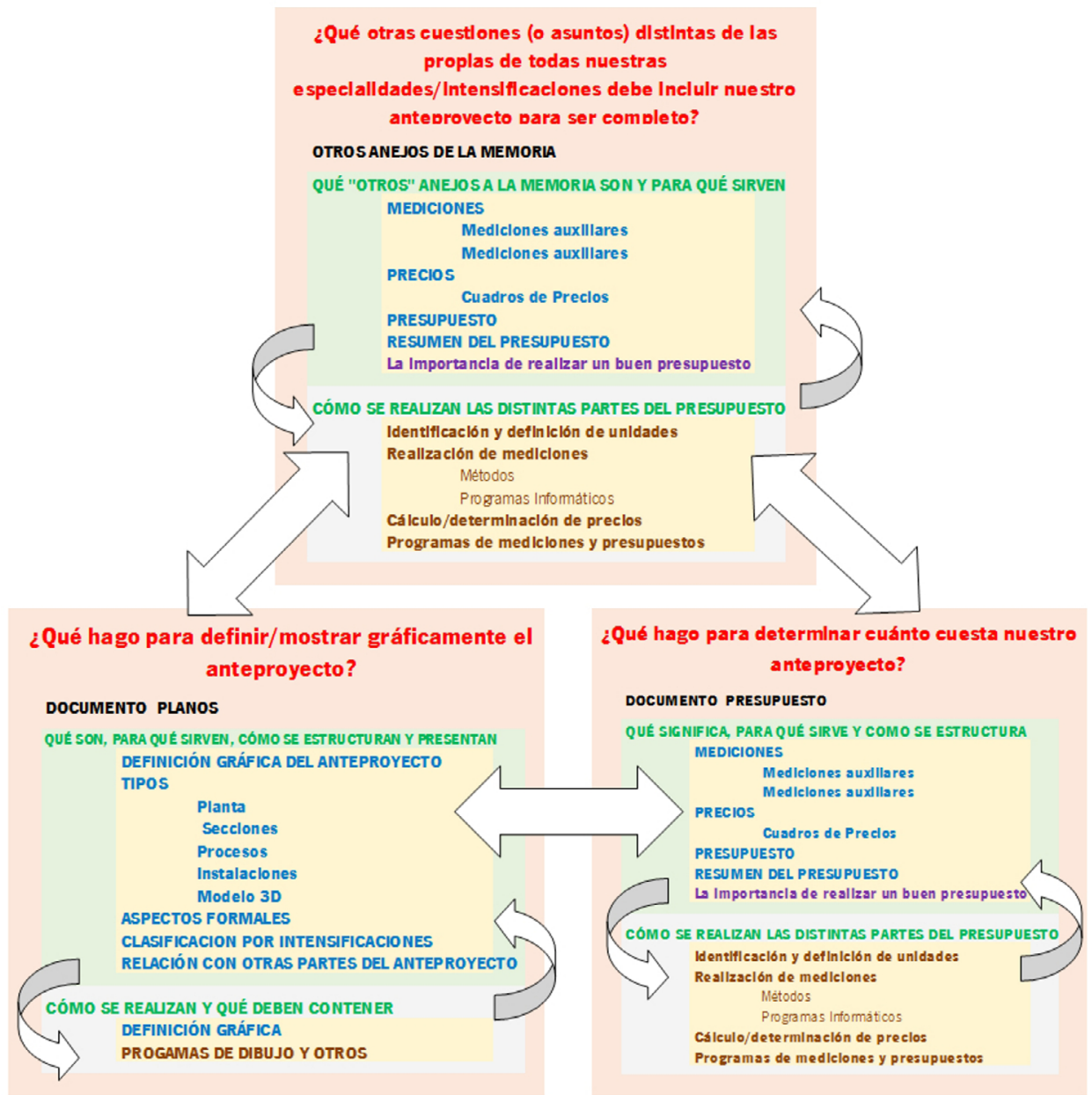

\section{Conceptos}

Procedimientos intelectuales

Actitudes/Valores

Figura 1. Mapa de contenidos y Problemas 


\section{Modelo metodológico posible}

En el modelo metodológico diseñado el alumno es el protagonista. Para su diseño me he basado en los conceptos: Reconfigurar el aula: quitar a la profesora del centro y Poner los alumnos a trabajar del libro Dar clase con la boca cerrada (Finkel, D. 2008), y requiere de un alto grado de compromiso, dedicación y responsabilidad por parte de los alumnos.

En el modelo metodológico se distinguen cinco actividades que se van desarrollando de manera cíclica tal y como se indica en la siguiente figura:

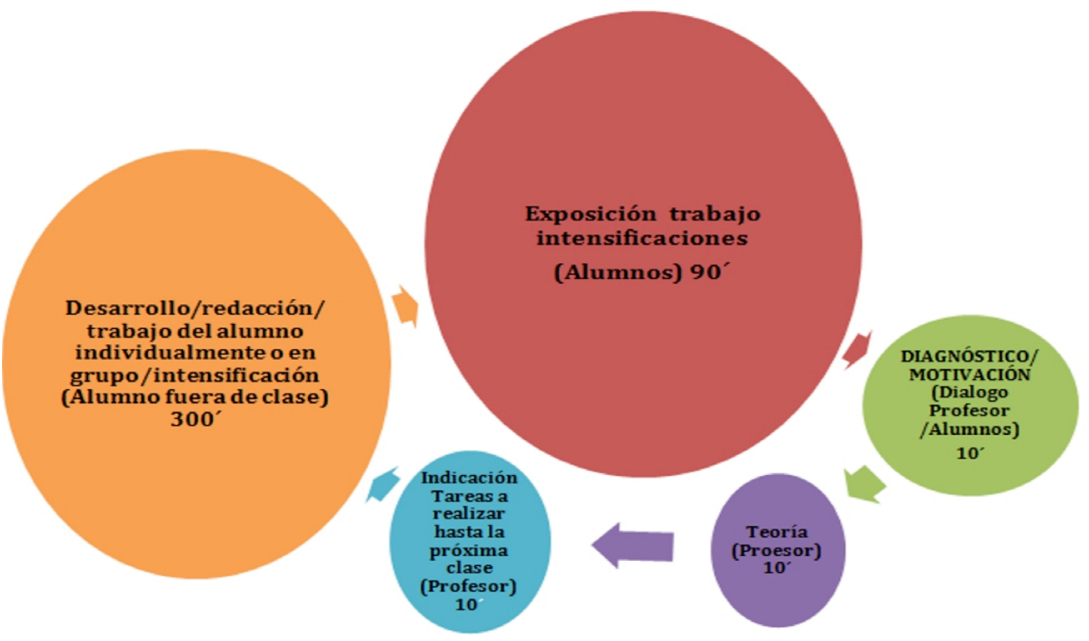

Figura 2. Modelo Metodológico posible

\section{Secuencia de actividades programada}

Se indican a continuación las fichas de las actividades programadas para cada uno de los tres bloques de contenidos del 2o CMD. En dichas fichas, además y por cuestiones de espacio, indico en color azul cómo se desarrollaron y ocurrieron realmente.

a) Primer bloque de contenidos: ¿Qué otras cuestiones distintas de las propias de todas nuestras 
Secuencia de actividades del primer bloque de contenidos del ciclo de mejora

\begin{tabular}{|c|c|c|}
\hline & & \\
\hline \multicolumn{3}{|c|}{$\begin{array}{l}\text { PARA QUÉ SIRVEN } \\
\text { s trabajos a reali- } \\
\text { e caso se trata de } \\
\text { idad y Salud, Con- } \\
\text { enido de los otros } \\
\text { on el resto de los } \\
\text { tiempo que tienen }\end{array}$} \\
\hline
\end{tabular}

Recursos: Intervención del profesor. Esquemas que se van construyendo/ desarrollando en la pizarra

\begin{tabular}{|c|c|c|}
\hline № 2 & $\begin{array}{l}\text { lizar durante la semana } \\
\text { hasta la próxima clase }\end{array}$ & Tiempo: $10^{-}$ \\
\hline \multicolumn{3}{|c|}{$\begin{array}{l}\text { Descripción: } \\
\text { CÓMO HACERLOS. INDICACIONES PARA LA PRÓXIMA EXPOSICIÓN DE GRUPOS } \\
\text { Interviene el profesor y expone concretamente como deben realizar los } \\
\text { otros anejos a la Memoria y las tareas que deben realizar los alumnos en } \\
\text { grupos para la siguiente clase. (Cada grupo de especialidad debe presentar } \\
\text { al resto el avance de su trabajo). } \\
\text { Esta actividad se desarrolló tal y como estaba prevista. Destaco la parti- } \\
\text { cipación de los alumnos ya que preguntaron todo lo que quisieron hasta } \\
\text { que les quedaron claras las actividades que tenían que realizar. }\end{array}$} \\
\hline
\end{tabular}

Recursos: Intervención del profesor. Esquemas que se van construyendo/ desarrollando en la pizarra

\begin{tabular}{|c|c|c|}
\hline No 3 & $\begin{array}{c}\text { Desarrollo/redacción/ tra- } \\
\text { bajo del alumno individual- } \\
\text { mente o en grupo } \\
\text { (Alumno fuera de clase) }\end{array}$ & Tiempo: $300^{-}$ \\
\hline
\end{tabular}

Jornadas de Formación e Innovación Docente del Profesorado | № 1 (2018) Esta obra se distribuye con la licencia Creative Commons Reconocimiento-NoComercial-SinObraDerivada $\quad 4.0$ Internacional (CC BY-NC-ND 4.0.) 


\section{Descripción:}

REDACCIÓN DE LOS OTROS ANEJOS A LA MEMORIA

Los alumnos realizan el trabajo fuera de clase de manera individual y también en.

A la vista de las presentaciones que realizaron los alumnos (ver actividades siguientes), entiendo que esta actividad, que se realizó en casa, se desarrolló correctamente.

Recursos: Publicaciones, Manuales, Catálogos, Normativa y Legislación, Aplicaciones informáticas

\begin{tabular}{|c|c|}
\hline № 4 & $\begin{array}{c}\text { Exposición trabajo de } \\
\text { especialidad }\end{array}$ \\
\hline
\end{tabular}

\section{Descripción:}

PRESENTACIÓN DE OTROS ANEJOS A LA MEMORIA REDACTADOS

Exposición del trabajo realizado y a realizar por los distintos grupos. (Hay

5)

Los distintos grupos presentarán (en 18') lo siguiente:

- cómo han desarrollado y cómo van a concluir sus otros anejos a la Memoria, mediante un índice o partes a desarrollar que deben explicar brevemente

- las dificultades o datos que necesitan y como lo han solventado o lo van a solventar

- la planificación de trabajos que van a realizar y compromiso de cumplimiento.

A pesar de que el profesor no pudo estar presente durante los primeros 45, la actividad se desarrolló tal y como estaba previsto, haciéndose realidad el concepto de Reconfigurar el aula: Quitar a la profesora del centro del libro: Dar clase con la boca cerrada (Finkel, D. 2008).

Recursos: Presentación de los alumnos con power point

\begin{tabular}{l|l|c|}
\hline \multicolumn{1}{|c|}{ No 5} & \multicolumn{1}{|c|}{ Diagnóstico y Motivación } & \multicolumn{1}{c|}{ Tiempo: $10^{-}$} \\
\hline Descripción: \\
DIAGNÓSTICO/DISCUSIÓN SOBRE OTROS ANEJOS A LA MEMORIA \\
REDACTADOS \\
El profesor a la vista de las exposiciones realizadas diagnostica la situa- \\
ción global y particular de cada grupo (si está bien enfocado, estructurado \\
y planificado el trabajo, si el avance es adecuado,...) e indica las acciones a \\
realizar para corregir las posibles desviaciones. \\
El profesor motiva a los alumnos para el alcance de los objetivos. \\
Esta actividad se desarrolló tal y como estaba prevista. En general el tra- \\
bajo de los alumnos estaba bien enfocado, estructurado y planificado y el \\
avance era adecuado. Intuía/percibía que el aprendizaje de los alumnos se \\
estaba desarrollando de manera adecuada.
\end{tabular}

Recursos: Intervención del profesor. Esquemas que se van construyendo/ desarrollando en la pizarra 
b) Segundo bloque de contenidos: ¿Qué hago para definir/mostrar gráficamente el anteproyecto?

\section{Tabla 2}

Secuencia de actividades del segundo bloque de contenidos del ciclo de mejora

\begin{tabular}{|c|c|c|}
\hline & & \\
\hline \multicolumn{3}{|c|}{$\begin{array}{l}\text { NOS DEL ANTE- } \\
\text { tos formales) } \\
\text { abajos a reali- } \\
\text { aso se trata de } \\
\text { do y para qué } \\
\text { formales, su } \\
\text { n con el resto } \\
\text { del tiempo que }\end{array}$} \\
\hline
\end{tabular}

Recursos: Intervención del profesor. Esquemas que se van construyendo/ desarrollando en la pizarra

\begin{tabular}{|c|c|c|}
\hline № 2 & $\begin{array}{l}\text { Indicación de tareas a reali- } \\
\text { zar durante la semana hasta } \\
\text { la próxima clase }\end{array}$ & Tiempo: $10^{-}$ \\
\hline \multicolumn{3}{|c|}{$\begin{array}{l}\text { Descripción: } \\
\text { CÓMO SE REALIZAN Y QUÉ DEBEN CONTENER. INDICACIONES PARA PRÓXIMA } \\
\qquad \text { EXPOSICIÓN DE GRUPOS } \\
\text { Interviene el profesor y expone concretamente como deben realizar los } \\
\text { Planos del Anteproyecto y las tareas que deben realizar los alumnos en } \\
\text { grupos para la siguiente clase. } \\
\text { (Cada grupo de especialidad debe presentar al resto el avance de su } \\
\text { trabajo). } \\
\text { Esta actividad se desarrolló tal y como estaba prevista. }\end{array}$} \\
\hline \multicolumn{3}{|c|}{$\begin{array}{l}\text { Recursos: Intervención del profesor. Esquemas que se van construyendo/ } \\
\text { desarrollando en la pizarra }\end{array}$} \\
\hline № 3 & $\begin{array}{c}\text { Desarrollo/redacción/ tra- } \\
\text { bajo del alumno individual- } \\
\text { mente o en grupo } \\
\text { (Alumno fuera de clase) }\end{array}$ & Tiempo: $300^{-}$ \\
\hline
\end{tabular}

Jornadas de Formación e Innovación Docente del Profesorado | № 1 (2018) Esta obra se distribuye con la licencia Creative Commons Reconocimiento-NoComercial-SinObraDerivada $\quad 4.0$ Internacional (CC BY-NC-ND 4.0.) 


\section{Descripción:}

REDACCIÓN DEL DOCUMENTO PLANOS

Los alumnos realizan el trabajo fuera de clase de manera individual y también en grupo.

A la vista de las presentaciones que realizaron los alumnos (ver actividades siguientes), entiendo que esta actividad, que se realizó realizada en casa, se desarrolló correctamente.

Recursos: Manuales, Aplicaciones informáticas

\begin{tabular}{|c|c|c|}
\hline 의 4 & & $90^{\circ}$ \\
\hline \multicolumn{3}{|c|}{$\begin{array}{l}\text { PLANOS } \\
\text { y a realizar por los distintos grupos. } \\
\text { in (en } 18^{\prime} \text { ) lo siguiente: } \\
\text { van a concluir sus Planos, mediante un ín- } \\
\text { deben explicar brevemente } \\
\text { cesitan y como lo han solventado o lo van } \\
\text { que van a realizar y compromiso de } \\
\text { y como estaba prevista. Aunque algunos } \\
\text { del que tenían disponible, finalmente se } \\
\text { de tiempo con las presentaciones de otros } \\
\text { dd en el tiempo disponible. }\end{array}$} \\
\hline
\end{tabular}

Recursos: Presentación de los alumnos con power point

\begin{tabular}{|l|l|l|}
\hline \multicolumn{1}{|c|}{ No $\mathbf{5}$} & \multicolumn{1}{|c|}{ Diagnóstico y Motivación } & Tiempo: $10^{-}$ \\
\hline Descripción: & DIAGNÓSTICO/DISCUSIÓN SOBRE EL DOCUMENTO PLANOS PRESENTADO \\
El profesor a la vista de las exposiciones realizadas diagnostica la situa- \\
ción global y particular de cada grupo (si está bien enfocado, estructurado \\
y planificado el trabajo, si el avance es adecuado,...) e indica las acciones \\
a realizar para corregir las posibles desviaciones. \\
El profesor motiva a los alumnos para el alcance de los objetivos. \\
Esta actividad se desarrolló tal y como estaba prevista. En general el tra- \\
bajo de los alumnos estaba bien enfocado, estructurado y planificado y el \\
avance era adecuado. Intuía/percibía que el aprendizaje de los alumnos \\
se estaba desarrollando de manera adecuada. \\
\hline Recursos: Intervención del profesor. Esquemas que se van construyendo/ \\
desarrollando en la pizarra
\end{tabular}

c) Tercer bloque de contenidos: ¿Qué hago para determinar cuánto cuesta nuestro anteproyecto? 
Secuencia de actividades del tercer bloque de contenidos del ciclo de mejora

\begin{tabular}{|c|c|c|}
\hline № 1 & eoría & o: $10^{-1}$ \\
\hline \multicolumn{3}{|c|}{$\begin{array}{l}\text { Descripción: } \\
\text { QUÉ SIGNIFICA, PARA QUÉ SIRVE EL PRESUPUESTO Y COMO SE ESTRUCTURA } \\
\text { Les describo y explico el contenido y planificación de los trabajos a realizar } \\
\text { en la siguiente fase o parte del anteproyecto. En este caso se trata del Pre- } \\
\text { supuesto del Anteproyecto. } \\
\text { Mi objetivo es conseguir que sean conscientes del contenido del Presu- } \\
\text { puesto del Anteproyecto, qué significa, para qué sirve, como se estructura y } \\
\text { su importancia y relación con el resto de los documentos del Anteproyecto, } \\
\text { del trabajo a realizar, del tiempo que tienen que realizarlo y de que pueden } \\
\text { realizarlo. } \\
\text { Esta actividad se desarrolló tal y como estaba prevista. }\end{array}$} \\
\hline
\end{tabular}

Recursos: Intervención del profesor. Esquemas que se van construyendo/ desarrollando en la pizarra

№ 2 Indicación de tareas a realizar durante la semana hasta la próxima

Tiempo: $10^{-}$ clase

\section{Descripción:}

CÓMO SE REALIZAN LAS DISTINTAS PARTES DEL PRESUPUESTO. INDICACIONES LA PRÓXIMA EXPOSICIÓN DE GRUPOS

Interviene el profesor y expone concretamente como deben realizar las distintas partes del presupuesto y las tareas que deben realizar los alumnos en grupos para la siguiente clase.

(Cada grupo de especialidad debe presentar al resto el avance de su trabajo).

Esta actividad se desarrolló tal y como estaba prevista.

Recursos: Intervención del profesor. Esquemas que se van construyendo/ desarrollando en la pizarra

\begin{tabular}{|c|c|c|}
\hline № 3 & $\begin{array}{c}\text { Desarrollo/redacción/ trabajo } \\
\text { del alumno individualmente o en } \\
\text { grupo/intensificación (Alumno } \\
\text { fuera de clase) }\end{array}$ & Tiempo: $300^{-}$ \\
\hline \multicolumn{3}{|c|}{$\begin{array}{l}\text { Descripción: } \\
\text { REDACCIÓN DE LOS DOCUMENTOS DEL PRESUPUESTO } \\
\text { LoS alumnos realizan el trabajo fuera de clase de manera individual y tam- } \\
\text { bién en grupo. } \\
\text { A la vista de las presentaciones que realizaron los alumnos (ver actividades } \\
\text { siguientes), entiendo que esta actividad, que se realizó realizada en casa, se } \\
\text { desarrolló correctamente. }\end{array}$} \\
\hline
\end{tabular}

Jornadas de Formación e Innovación Docente del Profesorado | № 1 (2018) Esta obra se distribuye con la licencia Creative Commons Reconocimiento-NoComercial-SinObraDerivada $\quad 4.0$ Internacional (CC BY-NC-ND 4.0.) 
Recursos: Libros, Manuales, Bancos de precios, Aplicaciones informáticas № 4 Exposición trabajo de especialidad

Tiempo: $90^{-}$

\section{Descripción:}

PRESENTACIÓN DE LOS DOCUMENTOS DEL PRESUPUESTO

Exposición del trabajo realizado y a realizar por los distintos grupos.

Los distintos grupos presentarán (en 18') lo siguiente:

cómo han desarrollado y cómo van a concluir su parte del presupuesto, mediante un índice o partes a desarrollar que deben explicar brevemente

las dificultades o datos que necesitan y como lo han solventado o lo van a solventar

la planificación de trabajos que van a realizar y compromiso de cumplimiento.

Esta actividad se desarrolló tal y como estaba prevista. Aunque algunos grupos emplearon más tiempo del que tenían disponible, finalmente se compensó este mayor empleo de tiempo con las presentaciones de otros grupos y se concluyó la actividad en el tiempo disponible.

Recursos: Presentación de los alumnos con power point

№ 5

Diagnóstico y Motivación

Tiempo: 10

\section{Descripción:}

DIAGNÓSTICO/DISCUSIÓN SOBRE EL PRESUPUESTO PRESENTADO

El profesor a la vista de las exposiciones realizadas diagnostica la situación global y particular de cada grupo (si está bien enfocado, estructurado y planificado el trabajo, si el avance es adecuado,...) e indica las acciones a realizar para corregir las posibles desviaciones.

El profesor motiva a los alumnos para el alcance de los objetivos.

Esta actividad se desarrolló tal y como estaba prevista. En general el trabajo de los alumnos estaba bien enfocado, estructurado y planificado y el avance era adecuado. Intuía/percibía que el aprendizaje de los alumnos se estaba desarrollando de manera adecuada.

Recursos: Intervención del profesor. Esquemas que se van construyendo/ desarrollando en la pizarra

\section{Cuestionario inicial-final}

Coincidiendo con los problemas-preguntas del mapa de contenidos, el cuestionario que se utilizó para hacer el seguimiento de la evolución de los estudiantes fue:

Pregunta 1: ¿Qué hago para determinar cuánto cuesta nuestro anteproyecto?

Pregunta 2: ¿Qué hago para definir/mostrar gráficamente el anteproyecto? 
Pregunta 3: ¿Qué otras cuestiones (o asuntos) distintas de las propias de todas nuestras especialidades/intensificaciones debe incluir nuestro anteproyecto para ser completo?

\section{Aplicación del CMD}

\section{Relato resumido de las sesiones.}

En general el resultado ha sido satisfactorio ya que se consiguieron los objetivos.

Las actividades se desarrollaron de manera normal, en el tiempo que estaba previsto y sin forzarlas. Solamente hubo algunas presentaciones de grupos que duraron más de lo previsto; pero se compensaron con las presentaciones de otros grupos. Esto se ha producido de manera natural.

La participación de los alumnos fue muy buena, realizaron correctamente el trabajo/actividades fuera del aula/en casa y prepararon sus intervenciones. Eso es lo que motivó que el resultado fuese satisfactorio; ya que el peso fundamental de las clases es la intervención de los alumnos.

En general el trabajo de los alumnos estuvo bien enfocado, estructurado y planificado y el avance fue adecuado. Intuía/percibía que el aprendizaje de los alumnos se estaba desarrollando de manera adecuada.

En cuanto a los detalles y conductas más significativos y las emociones sentidas, tanto positivas como negativas, indicar que no estoy sorprendido por el buen comportamiento y actitud de los alumnos: lo esperaba. Fueron responsables, participativos, activos, diligentes, se comunicaron e interrelacionaron muy bien y se interesaron por el desarrollo de la asignatura. Yo terminé muy satisfecho del segundo ciclo de mejora y disfruté mucho con él. Pienso que para los alumnos fue una buena experiencia y sacaron buen provecho de la misma. 


\section{Evaluación del aprendizaje de los estudiantes. Escaleras de Aprendizaje.}

Antes de iniciar el ciclo de mejora docente y después de finalizar el mismo los 20 alumnos del curso cumplimentaron el cuestionario indicado anteriormente. A partir de dicho cuestionario y para cada una de sus preguntas se confeccionaron las escaleras de aprendizaje. Dichas escaleras se corresponden con las figuras no3, 4 y 5 que se incluyen a continuación. En ellas, los porcentajes de menor tamaño corresponden a las respuestas iniciales al cuestionario y los de mayor tamaño y en rojo a las respuestas finales al mismo.

Pregunta 1: ¿Qué hago para determinar cuánto cuesta nuestro anteproyecto?

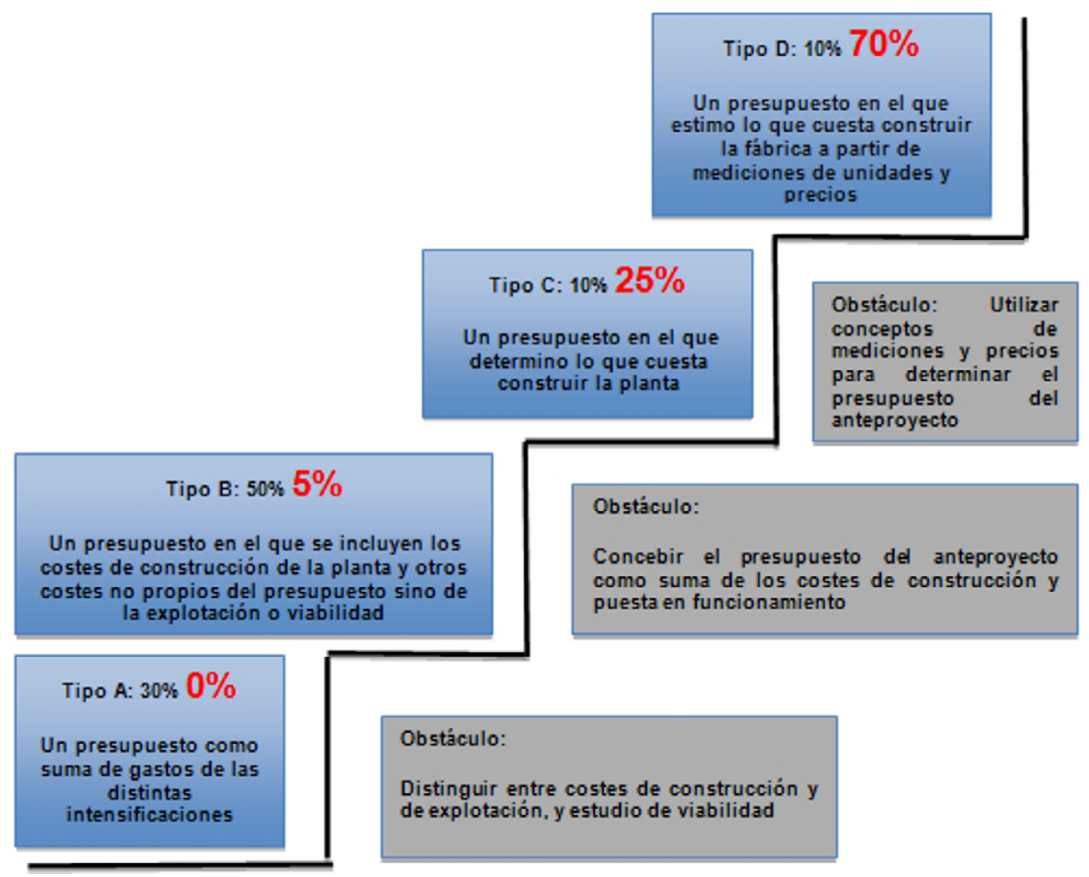

Figura 3. Escalera de aprendizaje correspondiente a la pregunta 1

Pregunta 2: ¿Qué hago para definir/mostrar gráficamente el anteproyecto?

Jornadas de Formación e Innovación Docente del Profesorado I № 1 (2018) Reconocimiento-NoComercial-SinObraDerivada $\quad 4.0$ Internacional (CC BY-NC-ND 4.0.) 

caracteristica de la instalación y esquemas de funcionamiento de las instalaciones y modelo 3D

Tipo B: $25 \% 0 \%$

Esquemas de funcionamiento de la instalación
Obstáculo:

Distintos tipos de planos (situación,

emplazamiento, plantas, alzados, detalles mant $^{\prime}$

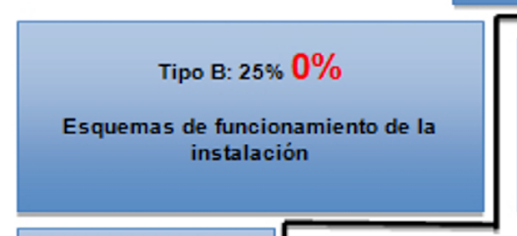

Obstáculo:

Concepto de planos para definición gráfica del anteproyecto

Tipo A: $10 \%, 0 \%$

Power Point

Figura 4. Escalera de aprendizaje correspondiente a la pregunta 2gunta 2.jpg Pregunta 3: ¿Qué otras cuestiones (o asuntos) distintas de las propias de todas nuestras especialidades/intensificaciones debe incluir nuestro anteproyecto para ser completo?

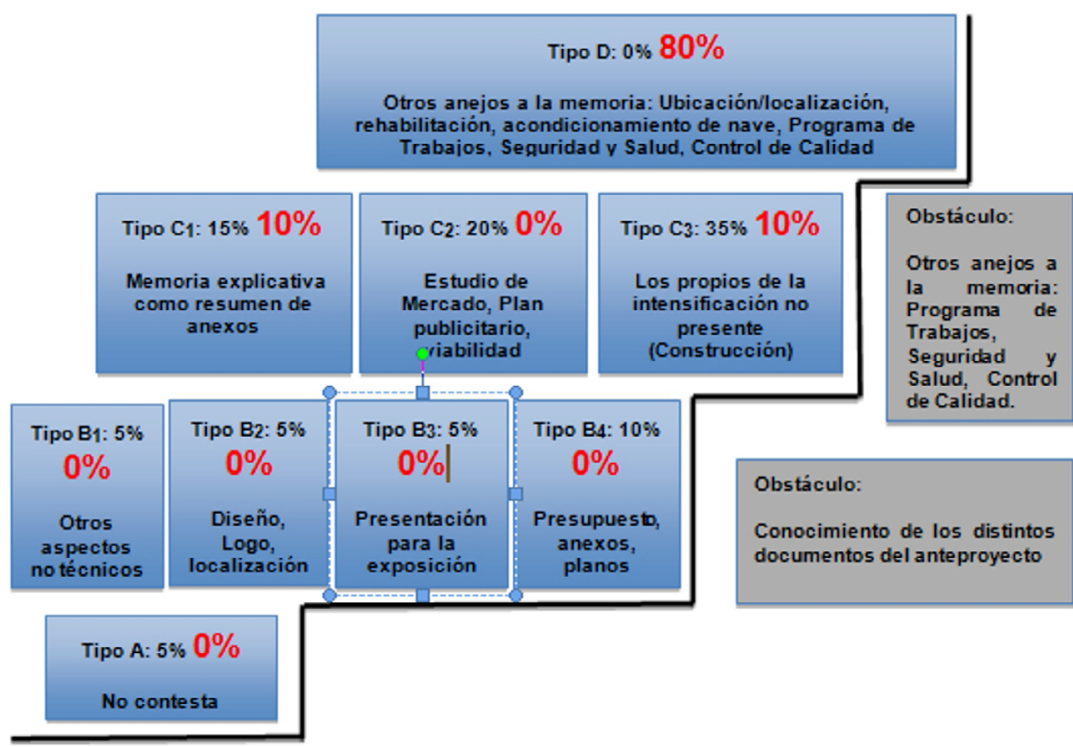

Figura 5. Escalera de aprendizaje correspondiente a la pregunta3

Jornadas de Formación e Innovación Docente del Profesorado | № 1 (2018)

(c) (i) E Esta obra se distribuye con la licencia Creative Commons Reconocimiento-NoComercial-SinObraDerivada Internacional (CC BY-NC-ND 4.0.) 
Analizando las escaleras de aprendizaje podemos observar lo siguiente:

En la pregunta no1 se ha pasado de una situación inicial en la que la mayoría de los alumnos estaban en los niveles más bajos (niveles A y B en los que estaban el $30 \%$ y el $50 \%$ de los alumnos respectivamente) a una situación final en la que todos los alumnos, salvo uno que está en el nivel B, están en los dos niveles más altos (niveles C y D en los que están el $25 \%$ y el $70 \%$ de los alumnos respectivamente).

En la pregunta no2 se ha pasado de una situación inicial en la que hay alumnos distribuidos en todos los niveles, a una situación final en la que todos los alumnos están en los dos niveles más altos (niveles C y D en los que están el $20 \%$ y el $80 \%$ de los alumnos respectivamente).

En la pregunta no3 se ha pasado de una situación inicial en la que había alumnos distribuidos sólo en los tres niveles más bajos, a una situación final en la que todos los alumnos están en los dos niveles más altos (niveles C y D en los que están $20 \%$ y el $80 \%$ de los alumnos respectivamente).

\section{Tabla de avances individuales}

También se confeccionó la tabla de avances individuales (Tabla 4), en la que para cada uno de los alumnos y para cada una de las preguntas del cuestionario se indica el nivel o escalón en el que se encontraba el alumno al inicio y al final de ciclo de mejora docente y la progresión que experimentó durante dicho ciclo. 
Tabla 4. Tabla de avances individuales

\begin{tabular}{|c|c|c|c|c|c|c|c|c|c|}
\hline \multicolumn{10}{|c|}{ NIVELES DE DESARROLLO DE IDEAS INICIALES Y FINALES } \\
\hline ALUMNO & \multicolumn{3}{|c|}{ PREGUNTA 1} & \multicolumn{3}{|c|}{ PREGUNTA 2} & \multicolumn{3}{|c|}{ PREGUNTA 3} \\
\hline & INI. & FIN. & & INI. & FIN. & & INI. & FIN. & \\
\hline 1 & C & D & +1 & C & D & +1 & $\mathrm{~B}_{4}$ & D & +2 \\
\hline 2 & B & B & 0 & C & D & +1 & $\mathrm{~B}_{4}$ & D & +2 \\
\hline 3 & A & C & +2 & A & C & +2 & $\mathrm{~B}_{1}$ & $\mathrm{D}$ & +2 \\
\hline 4 & $\mathrm{D}$ & D & 0 & B & C & +1 & $\mathrm{~B}_{3}$ & D & +2 \\
\hline 5 & B & D & +2 & A & D & +3 & $\mathrm{~B}_{3}$ & D & +2 \\
\hline 6 & A & D & +3 & C & D & +1 & $\mathrm{C}_{2}$ & $C_{1}$ & 0 \\
\hline 7 & B & D & +2 & C & D & +1 & $\mathrm{C}_{3}$ & D & +1 \\
\hline 8 & B & C & +1 & B & D & +2 & $\mathrm{C}_{3}$ & $C_{3}$ & 0 \\
\hline 9 & A & C & +2 & B & D & +2 & $\mathrm{C}_{3}$ & $\mathrm{C}_{3}$ & 0 \\
\hline 10 & $B$ & D & +2 & D & D & 0 & $C_{2}$ & D & +1 \\
\hline 11 & A & D & +3 & $C$ & D & +1 & $\mathrm{C}_{2}$ & D & +1 \\
\hline 12 & A & C & +2 & D & D & 0 & $C_{1}$ & $C_{1}$ & 0 \\
\hline 13 & B & D & +2 & C & D & +1 & $C_{3}$ & D & +1 \\
\hline 14 & $B$ & C & +1 & $C$ & D & +1 & $\mathrm{C}_{3}$ & D & +1 \\
\hline 15 & D & D & 0 & D & D & 0 & $C_{3}$ & D & +1 \\
\hline 16 & B & D & +2 & B & C & +1 & A & D & +3 \\
\hline 17 & C & D & +1 & D & D & 0 & $C_{2}$ & $\mathrm{D}$ & +1 \\
\hline 18 & A & D & +3 & $B$ & C & +1 & $\mathrm{C}_{3}$ & D & +1 \\
\hline 19 & B & D & +2 & C & D & +1 & $C_{1}$ & $\mathrm{D}$ & +1 \\
\hline 20 & B & D & +2 & $\mathrm{D}$ & D & 0 & $C_{1}$ & D & +1 \\
\hline
\end{tabular}

Analizando la tabla de avances individualizados podemos observar lo siguiente:

- En la pregunta no1 todos los alumnos han subido escalones (1, 2 ó 3 según el caso) salvo tres alumnos que se han mantenido en los niveles en los que estaban (dos alumnos en el nivel superior y un alumno en el segundo nivel).

- En la pregunta no2 todos los alumnos han subido escalones (1, 2 ó 3 según el caso) salvo tres alumnos 
que ya estaban en el nivel superior y se han mantenido en él.

- En la pregunta no3 todos los alumnos han subido escalones (1, 2 ó 3 según el caso) salvo cuatro alumnos que se han mantenido en el tercer nivel y no han alcanzado el último nivel.

A la vista de todo lo anterior (escaleras de aprendizaje y tabla de avances individuales), podemos concluir que el "aprendizaje se ha producido" ya que la mayor parte de los alumnos han evolucionado (escalado posiciones) y han alcanzado el máximo nivel de aprendizaje (el 70\%, 80\% y 80\% de los alumnos en las preguntas n으, n을 y no3 respectivamente han alcanzado el máximo nivel de aprendizaje).

\section{Evaluación del CMD}

\section{Cuestiones a mantener y cambios a introducir para un futuro Ciclo de Mejora más amplio}

Cuestiones a mantener:

a) Reconfigurar el aula: quitar al profesor del centro de atención del alumno y que ésta se centre en la materia sobre la que se esté trabajando

b) Poner a los estudiantes a trabajar

c) Conocer y analizar los modelos mentales de los estudiantes mediante cuestionarios y escaleras de aprendizaje

Cambios a introducir en un futuro

a) Introducir la evaluación del estudiante durante el proceso de enseñanza aprendizaje utilizando el portafolio

b) Introducir la evaluación del docente y del diseño didáctico durante el proceso mediante el 
diario del profesor y encuestas de opinión de los estudiantes.

\section{Aspectos de la experiencia que se pretenden incorporar a toda la práctica docente habitual}

a) Reconfigurar el aula: quitar al profesor del centro de atención del alumno y que ésta se centre en la materia sobre la que se esté trabajando

b) Poner a los estudiantes a trabajar

c) Conocer y analizar los modelos mentales de los estudiantes mediante cuestionarios y escaleras de aprendizaje

d) Evaluación del estudiante durante el proceso de enseñanza aprendizaje utilizando el portafolio

e) Evaluación del docente y del diseño didáctico durante el proceso mediante el diario del profesor y encuestas de opinión

\section{Principios didácticos que han guiado la experiencia y que deben permanecer en el futuro:}

- Deducidos de la experiencia y aprendizaje alcanzados en este seminario y basado en las lecturas del libro Dar clase con la boca cerrada (Finkel, D. 2008):

- El diseño de una experiencia que enseñe

- El diseño de una secuencia de preguntas que construyan el conocimiento/aprendizaje

- Convertir productos de conocimiento en procesos que conducen a ellos

- Convertir la experiencia en completa

- Reconfigurar el aula: quitar al profesor del centro de atención del alumno (esto se ha conseguido en este 2o CMD)

- Poner a los estudiantes a trabajar (esto se ha conseguido en este 2o CMD)

- Crear esquemas de aprendizaje 
- Deducidos de la experiencia y aprendizaje alcanzados en este seminario y basados en la lectura del libro Enseñanza Universitaria. Cómo mejorarla. (Porlán, R. 2017):

- Aportar las ayudas necesarias en el proceso de aprendizaje del alumno (mediante actividades y recursos específicos) para orientar la transición desde los modelos propios del alumno a los propios de la disciplina

- Conocer y analizar los modelos mentales del alumno mediante los cuestionarios y escaleras de aprendizaje

- La evaluación del estudiante durante el proceso de enseñanza-aprendizaje mediante el portafolio o carpetas de aprendizaje

- La evaluación del docente y del diseño didáctico durante el proceso mediante el diario del profesor y las encuestas de opinión

\section{Referencias Bibliográficas}

Finkel, D. (2008). Dar clase con la boca cerrada. Valencia: Publicaciones Universidad de Valencia.

Porlán, R. (2017). Enseñanza universitaria. Cómo mejorarla. Madrid: Ediciones Morata, S.L.

Jornadas de Formación e Innovación Docente del Profesorado | № 1 (2018) Esta obra se distribuye con la licencia Creative Commons 